\title{
Impacto del castor (Castor canadensis, Rodentia) en bosques de lenga (Nothofagus pumilio) de Tierra del Fuego, Chile
}

\author{
Impact of american beaver (Castor canadensis, Rodentia) in lenga (Nothofagus pumilio) \\ forests of Tierra del Fuego, Chile
}

\author{
Aida Baldini $\mathbf{U}^{\mathrm{a}}$, Juan Oltremari $\mathrm{A}^{\mathrm{b} *}$, Mauricio Ramírez \\ aCorporación Nacional Forestal, Programa Protección Fitosanitaria, Santiago, Chile. \\ *Autor de correspondencia: 'Pontificia Universidad Católica de Chile, Facultad de Agronomía e Ingeniería Forestal, \\ Departamento de Ciencias Forestales, Av. Vicuña Mackenna 4860, Santiago, Chile, joltrama@puc.cl \\ ${ }^{\mathrm{c} C E D R E M}$ Consultores, Santiago, Chile.
}

\begin{abstract}
SUMMARY
Castor canadensis is a North American rodent introduced in the Argentinean part of Tierra del Fuego in 1946, invading Chilean territories located in the same area. In this zone, not only do beavers build dikes, but they also feed on arboreal material, mainly from Nothofagus pumilio. This study tried to prove that damage on both biomass and volume in the N. pumilio forest is caused by beavers. The objectives of the study were: to identify the type of damage and to quantify the loss of wood biomass and gross volume of the most abundant forest species. Results indicated that more than half of measured trees presented some damage; the highest damage being presented by $N$. pumilio. Loss in biomass reaches almost $15 \mathrm{t} \mathrm{ha}^{-1}$; amount superior to results found in studies made on the endemic environment of beavers. In relation to wood volume, over half of the stocks of $N$. pumilio forests associated to water resources have been lost in Tierra del Fuego due to the effect of beaver. The most important volume loss of this species derives from the death of individuals in flooded zones as a result of dike construction.
\end{abstract}

Key words: Nothofagus pumilio, Castor canadensis, environmental damages, invading species.

\section{RESUMEN}

Castor canadensis es un roedor de Norteamérica introducido a la porción argentina de Tierra del Fuego en el año 1946, invadiendo territorios chilenos del mismo sector. En esta zona los castores construyen diques y se alimentan de material arbóreo extraído principalmente de Nothofagus pumilio. En el estudio se intentó probar que los castores provocan daño en la biomasa y volumen de los bosques de N. pumilio. Los objetivos del estudio fueron identificar los tipos de daños y cuantificar las pérdidas de biomasa leñosa y de volumen bruto de la especie forestal más abundante. Los resultados indicaron que más de la mitad de los árboles medidos presentó algún daño, correspondiendo en su gran mayoría a $N$. pumilio. Las pérdidas en biomasa alcanzaron casi $15 \mathrm{t}$ $\mathrm{ha}^{-1}$, cantidad superior a los resultados encontrados en estudios realizados en el ambiente endémico de los castores. Respecto a volumen de madera, más de la mitad de la existencia de bosques de $N$. pumilio asociados a cursos de agua ha sido dañada en Tierra del Fuego por efecto de los castores. La pérdida más importante de volumen de esta especie resulta de la muerte de individuos en zona de inundación de la represa castoril.

Palabras clave: Nothofagus pumilio, Castor canadensis, daños ambientales, especies invasoras.

\section{INTRODUCCIÓN}

Castor canadensis (Kuhl 1820, Rodentia) es un roedor perteneciente a la familia Castoridae, originario de Estados Unidos de Norteamérica y Canadá, encontrándose en cualquier lugar donde existan ríos y árboles deciduos, dentro de su rango de distribución, con habilidad de cortar árboles maduros para alimentación y construcción (Naiman et al. 1988, Nummi 2006). Los diques construidos por los castores cambian el régimen de descarga anual de un río, disminuyen la velocidad de la corriente, expanden la superficie de los suelos inundados, y aumentan la retención de sedimentos y materia orgánica (Naiman et al. 1988, Wright et al. 2002). La mayor retención de materia orgánica se explica porque los castores rara vez son capaces de consumir la totalidad del material vegetal caído (Rosell et al. 2005), generando hábitat para otras especies, particularmente herbáceas (Schlatter et al. 2002, Mella et al. 1995). Rosell et al. (2005) además destacan que no sólo existe una alteración directa sobre el flujo hídrico, sino también en la geomorfología por el propio efecto de los diques y los refugios que construyen para su supervivencia. Los castores también alteran la composición química de las aguas por la mayor sedimentación, así como su tem- 
peratura por la tala de árboles que proporcionan sombra, provocando alteraciones en la estructura y dinámica de los ecosistemas (Johnston y Naiman 1987).

Las alteraciones físicas que producen los castores en los ecosistemas tienen un "efecto cascada" en muchas otras especies, aunque estas consecuencias bióticas están menos caracterizadas. De acuerdo a Crooks (2002) es mucho más fácil medir el efecto sobre las propiedades de los ecosistemas que cuantificar las diferentes formas en que las especies pueden responder a esos cambios.

Según Fryxell y Doucet (1993), las especies arbóreas más preferidas por $C$. canadensis en su hábitat natural son Populus tremuloides Michx., Alnus rugosa (Du Roi) Clausen, y en parte Acer rubrum L., lo que indica una clara preferencia por especies de maderas blandas. El roedor fue introducido en el sector argentino de Tierra del Fuego en 1946 (Skewes et al. 2006), como una iniciativa para generar recursos peleteros (Anderson et al. 2006a), e ingresó a territorio chileno en el área correspondiente a la ribera noroeste del lago Fagnano (aproximadamente $54^{\circ}$ 30' S), provincia de Tierra del Fuego (Lizarralde 1993, Lizarralde et al. 2004, Wallem et al. 2007). Skewes et al. (2006) estiman que la población de castores en territorio chileno de Tierra del Fuego, incluyendo también Isla Navarino, alcanza a 61.300 individuos, expandiéndose a una tasa linear de 2,3 a $6,3 \mathrm{~km}$ al año, por falta de predadores, en combinación con una alta tasa de reproducción (Anderson et al. 2006b). En esta zona los castores construyen diques y se alimentan de material arbóreo extraído principalmente de Nothofagus pumilio (Poepp. et Endl.) Krasser, la especie más abundante. También utilizan especies forestales menos abundantes en Tierra del Fuego, como Nothofagus antarctica (Forst.) Oerst. y en parte Nothofagus betuloides (Mirb.) Oerst. (Siefeld y Venegas 1980, Skewes et al. 1999, Wallem et al. 2007), todas especies pertenecientes a la masa boscosa aledaña a los cursos de agua. También suele atacar a Barberis spp y otras especies arbustivas (Mella et al. 1995, Anderson et al. 2006b).

La acción de los castores es restringida a una franja relativamente estrecha de bosque alrededor del régimen hídrico, por lo tanto, el impacto se concentra dentro de numerosas áreas de reducida superficie, pero que en su conjunto producen un alto impacto ambiental (Martínez et al. 2006, Johnston y Naiman 1987). Este impacto resulta relevante por la ausencia de una historia evolutiva entre los castores y los bosques de Nothofagus spp. Se trata de bosques que carecen de los mecanismos defensivos y estrategias reproductivas que se encuentran en los bosques de su ambiente natural (Anderson et al. 2006a).

En Tierra del Fuego la construcción de diques ha modificado el hábitat en grandes extensiones de terreno debido a la inundación de bosques, principalmente de $N$. pumilio, y a la desviación de ríos. Se menciona que $C$. canadensis ha persistido en la construcción de represas de hasta 1,5 m de altura y $100 \mathrm{~m}$ de longitud, además de la construcción de extensos canales a lo largo de laderas boscosas, por donde transportan fácilmente su alimento (Iriarte 2000). Esta invasión es analizada en forma exhaustiva por Jaksic et al. (2002), quienes corroboran que los sectores con mayor daño corresponden a bosques de protección para el régimen hídrico, especificados por la legislación forestal vigente, que expresamente protege al arbolado situado a menos de $200 \mathrm{~m}$ desde las orillas de ríos y vertientes. Coincide también con terrenos de la Wildlife Conservation Society (WCS), declarados por dicho organismo como área protegida privada (Parque Karukinka).

No obstante los daños ambientales producidos por los castores, el efecto sobre la biomasa forestal ha sido poco cuantificado en Chile, y las investigaciones se han centrado más bien en elementos cualitativos. Skewes et al. (1999) confirman que los castores tienen mayor preferencia por ambientes donde conviven las especies $N$. pumilio y $N$. antarctica, asociados a cursos de agua. En ese estudio ha quedado demostrado que los únicos hábitat que son ocupados en menor proporción por los castores son aquellos ubicados en el límite altitudinal de la vegetación, además de algunos sectores de bosques puros de $N$. betuloides, especie que posee una madera más densa que $N$. pumilio.

En otros países se han logrado mayores avances cuantitativos en la pérdida de biomasa. En un reciente estudio en territorios argentinos de Tierra del Fuego, Martínez et al. (2006) concluyen que la acción de los castores altera la dinámica de la vegetación, modificando la biomasa y la composición de las comunidades originales. Los autores destacan que la mantención de estas colonias de castores no es sustentable, principalmente debido a que los bosques de Nothofagus spp. no están adaptados a soportar el impacto de los castores por un tiempo prolongado, que por su escasa regeneración transforman su ecosistema original de un bosque primario cerrado a un ambiente de praderas abiertas. Esta escasez reproductiva se ve agravada aún más por el ramoneo de la regeneración temprana de $N$. pumilio por parte de Lama guanicoe (Artiodactyla: Camelidae) (Pulido et al. 2000).

Härkönen (1999) presenta una evaluación del daño causado por $C$. canadensis en bosques del centro-sur de Finlandia, considerando las características de las áreas dañadas, tipo y severidad del daño. Las principales especies de los bosques analizados son Pinus sylvestris L., Picea abies (L.) Karst. y Betula spp., donde P. sylvestris es la especie dominante. Por su parte Johnston y Naiman (1990) presentan un estudio realizado en Amity Creek, Minnesota, Estados Unidos, en el cual cuantifican la biomasa leñosa removida por efecto de los castores, y también determinan cómo la herbivoria selectiva afecta la densidad, área basal y composición de especies en un bosque ribereño.

El presente estudio pretende demostrar que los castores provocan cuantiosos daños por pérdida de biomasa y volumen en los bosques de $N$. pumilio de Tierra del Fuego, en terrenos legalmente definidos como de protección. Considerando el evidente impacto ambiental de los 
castores en los bosques nativos de Tierra del Fuego, los objetivos del estudio son: (1) determinar el tipo de daño en los bosques afectados por $C$. canadensis, (2) cuantificar la biomasa leñosa por hectárea removida por $C$. canadensis de la especie más abundante (N. pumilio) y (3) cuantificar las pérdidas de volumen bruto de la principal especie forestal del área de estudio (N. pumilio).

\section{MÉTODOS}

Área de estudio, muestreo y mediciones realizadas. Para la cuantificación de la pérdida de biomasa y volumen bruto sólo se consideró la especie $N$. pumilio debido a su mayor abundancia relativa y, por ello, constituir la especie forestal más afectada por $C$. canadensis en Tierra del Fuego, conforme a los abundantes antecedentes bibliográficos sobre este aspecto (Wallem et al. 2007).

El hábitat de los castores en Tierra del Fuego corresponde a una estrecha banda de bosques aledaña a cursos de agua. Debido a que el bosque en esa zona del país se concentra en el sector sur, todo el régimen hídrico del sector sur de Tierra del Fuego incluye áreas potenciales de muestreo, y fue donde se concentró el presente estudio. Según Gajardo (1993), el sector sur está dominado por bosques de N. pumilio-N. antarctica, y en las cercanías al nivel del mar domina $N$. betuloides.

Dentro del sector sur de Tierra del Fuego se trazó un eje en dirección sureste-noroeste, el cual cubre las principales redes hidrográficas asociadas a bosques. A lo largo de este eje, se definieron tres sectores circulares de 10.000 ha: Pampa Guanaco, en la ribera este del lago Blanco (sector I), lago Lynch, en su ribera noroeste (sector II) y Timaukel, que corresponde a los bosques más septentrionales de Tierra del Fuego (sector III) (figura 1).

Para seleccionar las áreas de muestro se utilizaron mapas digitalizados pertenecientes a Wildlife Conservation Society (WCS), entidad que desarrolla actividades de investigación en Tierra del Fuego, y fueron desplegados en ArcView GIS 3.2. Sobre esta base se identificaron todos los bosques ribereños a menos de $100 \mathrm{~m}$ del eje del curso de agua, hacia ambas riberas (denominados buffers), siguiendo las propuestas de Härkönen (1999) y los resultados de Barnes y Dibble (1988). Se utilizó la distancia de $100 \mathrm{~m}$ debido a que los estudios en Tierra del Fuego, así como en otros lugares, indican que es la máxima distancia registrada de adentramiento de los castores (Briones et al. 2001, Gallant et al. 2004, Lizarralde et al. 2004).

La superficie de todos los buffers en el sector sur de Tierra Fuego alcanzó a 33.828 ha y es la máxima superficie de bosques asociados a cursos de agua a menos de $100 \mathrm{~m}$, correspondiendo al área potencial con presencia de actividad de castores. Otros estudios de distribución de $C$. canadensis en la misma área subestiman esta superficie al considerar una menor distancia de cursos de agua (Olave y Skewes 2000).
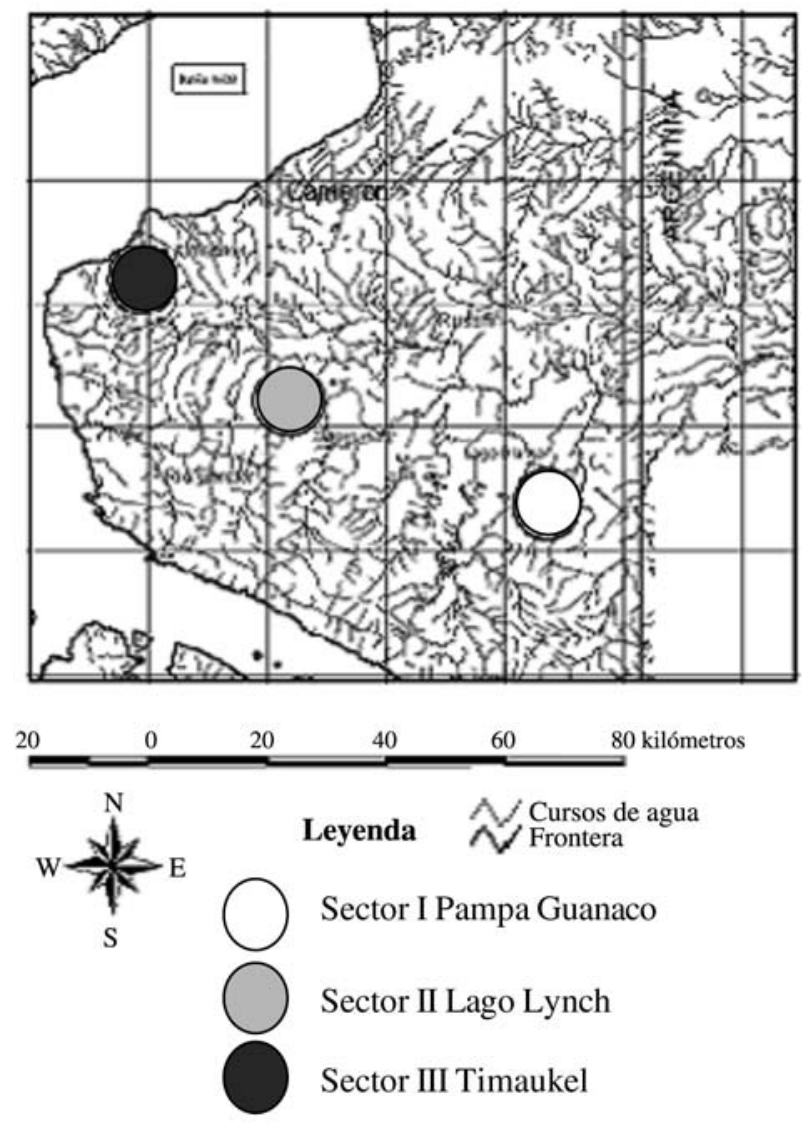

Figura 1. Sectores de muestreo.

Sampling areas.

Para determinar áreas de muestreo para cada sector se identificaron puntos con sus respectivas coordenadas UTM cada un kilómetro a lo largo de los buffers. En cada sector se realizó una selección aleatoria de siete áreas de muestreo (muestras), exportando al software MS Excel las coordenadas de todas las áreas de muestreo y aplicando la función "aleatorio". En cada una de estas siete áreas de muestreo, seleccionadas al azar, se establecieron tres parcelas circulares de $254 \mathrm{~m}^{2}$ en un transecto perpendicular al curso de agua, basado en el método de Johnston y Naiman (1990). Siguiendo este procedimiento el estudio incluyó un total de 63 parcelas.

Las mediciones dentro de cada parcela se realizaron durante los meses de diciembre de 2005 y enero de 2006 y fueron las siguientes:

- Árboles no talados ni dañados: especie, diámetro altura de pecho (DAP) y etapa de desarrollo.

- Árboles talados: especie, etapa de desarrollo, diámetro altura de corte, altura de corte, discriminando la tala de castores por la forma cónica del tocón, altura sobre el suelo, y las marcas de los dientes de $C$. canadensis.

- Árboles anillados o dañados: especie, etapa de desarrollo, altura de daño, diámetro de daño y DAP.

- Árboles muertos en zona de inundación: DAP de 10 árboles elegidos al azar. 
Análisis de datos. El tipo y frecuencia de daños es el resultado de las mediciones de las parcelas individuales y en este caso se consideró a las tres especies arbóreas presentes (N. pumilio, N. antarctica y $N$. betuloides). Para cuantificar la biomasa de $N$. pumilio removida por castores se utilizó un modelo logarítmico, desarrollado para Tierra del Fuego a partir de muestras en un bosque mixto natural de N. pumilio y N. betuloides (Magni 1995) [1].

$$
\text { Ln PTA }=-0,85+1,93 \text { Ln DAP }
$$

donde,

PTA: peso seco total del árbol $(\mathrm{kg})$.

DAP: diámetro a la altura del pecho $(\mathrm{cm})$.

Para determinar la pérdida de volumen bruto de $N$. pumilio por tala e inundación se utilizaron los modelos de Schmidt y Urzúa (1982) para bosques de $N$. pumilio del sector chileno de Tierra del Fuego. El volumen removido por tala se estimó a partir de tablas de rodal correspondientes a los mismos bosques medidos, proyectando los tocones talados por castores a su respectivo DAP y considerándolos como individuos pertenecientes al bosque, obteniendo así una simulación del bosque original (bosque hipotético).

En el caso específico de los árboles muertos por inundación los valores de volúmenes por hectárea para cada área de muestreo dependieron de la densidad de la parcela más cercana al curso de agua, ya que el bosque muerto en inundación es una verdadera radiografía del mismo bosque años antes. De este modo, las estimaciones correspondieron al volumen del árbol promedio encontrado en zona de inundación (diámetro promedio de 10 árboles seleccionados al azar en la zona de inundación), el cual fue multiplicado por la densidad antes mencionada para obtener el volumen por hectárea. En zonas inundadas, por condiciones de inaccesibilidad, debió descartarse el uso de parcelas de muestreo.

Debido a que para los árboles talados sólo se disponía del diámetro del tocón, se utilizaron los datos correspondientes a aquellos árboles que presentan anillamiento o descortezamiento, a los cuales se les midió el DAP y el diámetro a la altura del daño, en la parte inferior del daño. Con estos datos se ajustó un modelo lineal que permite utilizar el diámetro a la altura de tala para estimar el DAP del árbol talado que ya no existe. La técnica utilizada fue regresión lineal simple donde $\mathrm{X}=\mathrm{DAP}$ de los árboles anillados o descortezados, e $\mathrm{Y}=$ diámetro a la altura del daño en la parte inferior de los árboles anillados o descortezados. El modelo entrega una ecuación del siguiente tipo [2]:

$$
\mathrm{Y}=\mathrm{AX}+\mathrm{B}
$$

donde,

A y $\mathrm{B}=$ coeficientes que entrega el ajuste lineal.

$\mathrm{Y}=$ diámetro de tala.

$\mathrm{X}=\mathrm{DAP}$ del tocón que es estimado por el modelo lineal.
La principal variable para cuantificar biomasa y volumen de la vegetación arbórea removida por castores es el diámetro a la altura de pecho (DAP) de los árboles talados. La distribución de esta variable hizo necesario recurrir a estadística no paramétrica para comparar los resultados entre cada sector circular inspeccionado. Específicamente se empleó la prueba no paramétrica de Wilcoxon (Rius et al. 1999), la cual compara las medianas de dos conjuntos de datos, considerando una hipótesis de que las medianas son iguales o sus diferencias son iguales a cero.

Se descartaron otras opciones no paramétricas como la prueba de Kruskal Wallis, empleada para realizar un análisis de varianza. La prueba de Wilcoxon, en cambio, es la opción no paramétrica para una comparación de medias, en este caso medianas. Una comparación de medianas entrega mayor información, debido a que las diferencias que se reflejan en diámetros preferidos por castores provienen de diferencias naturales existentes en los diámetros de bosques de N. pumilio, producto de los variados índices de sitios existentes para los bosques de Tierra del Fuego, de acuerdo a Schmidt ${ }^{1}$. De esta manera, la prueba de Wilcoxon explica si las diferencias en los diámetros preferidos por castores son por azar o se deben a variaciones naturales en los bosques.

\section{RESULTADOS}

Tipo y frecuencia de daños. De los 864 árboles medidos, 371 (43\%) fueron talados y 63 (7\%) fueron anillados, mientras que $430(50 \%)$ no presentaron daños. De las especies dañadas, 313 (72\%) correspondieron a individuos de $N$. pumilio (cuadro 1), corroborando estudios anteriores que la destacan, debido a su abundancia relativa, como la especie más afectada por los castores en Tierra del Fuego. El tipo y frecuencia de daños demostró el alto impacto de las actividades de los castores en los bosques ribereños, alterando su estructura y dinámica natural.

Cuadro 1. Tipo de daño por especie forestal. Type of damage by tree species.

\begin{tabular}{llrrc}
\hline \multirow{2}{*}{ Sector } & Especie & \multicolumn{3}{c}{ Número de árboles } \\
\cline { 3 - 5 } & & Medidos & Talados & Anillados \\
\hline I & N. pumilio & 305 & 137 & 14 \\
\hline II & N. pumilio & 225 & 51 & 31 \\
& N. antarctica & 7 & 5 & 1 \\
\hline III & N. pumilio & 173 & 68 & 12 \\
& N. antarctica & 132 & 109 & 1 \\
& N. betuloides & 22 & 1 & 4 \\
\hline
\end{tabular}

1 H Schmidt. Profesor Titular, Facultad de Ciencias Forestales, Universidad de Chile. Comunicación personal, 2006. 
Distribución diamétrica de los árboles talados. El modelo lineal que utilizó el diámetro a la altura de tala para estimar el DAP del árbol talado, posibilitó obtener la distribución por clase diamétrica de la totalidad de los árboles talados (cuadro 2).

Cuadro 2. Distribución de los árboles talados por clase diamétrica.

Distribution of foraged trees by diametric class.

\begin{tabular}{cc}
\hline Clase DAP $(\mathrm{cm})$ & Número de árboles talados \\
\hline $2,5-7,5$ & 195 \\
$7,6-12,5$ & 79 \\
$12,6-17,5$ & 42 \\
$17,6-22,5$ & 20 \\
$22,6-27,5$ & 11 \\
$27,6-32,5$ & 9 \\
$32,6-37,5$ & 7 \\
$37,6-42,5$ & 2 \\
$42,6-47,5$ & 2 \\
$47,6-52,5$ & 3 \\
$52,6-57,5$ & 1 \\
\hline TOTAL & 371 \\
\hline Diámetro promedio $(\mathrm{cm})$ & 10,7 \\
\hline
\end{tabular}

El cuadro 2 evidencia una clara preferencia de parte de los castores por los diámetros menores. Casi el 53\% de los árboles talados pertenece a la primera clase diamétrica, es decir árboles con diámetros hasta 7,5 cm. Esta preferencia corrobora resultados anteriores del comportamiento de los castores en su ambiente endémico (Johnston y Naiman 1990).

Pérdida de biomasa leñosa. Considerando los tres sectores en conjunto, el promedio global de biomasa leñosa perdida fue de 14,9 $\mathrm{t} \mathrm{ha}^{-1}$. En el sector I (Pampa Guanaco) la biomasa removida de $N$. pumilio alcanzó una media de 21,6 $\mathrm{t} \mathrm{ha}^{-1} \mathrm{y}$ sólo las áreas de muestreo 2 y 4 no presentaron actividad de castores, y como consecuencia no se presentó biomasa leñosa removida. En el sector II (Lago Lynch) la biomasa media removida de $N$. pumilio fue inferior $\left(15,9 \mathrm{t} \mathrm{ha}^{-1}\right)$ y una de las áreas de muestreo no presentó tala, aunque existió actividad de castores. En el sector III (Timaukel) la biomasa media removida de $N$. pumilio fue sólo de $7,1 \mathrm{t} \mathrm{ha}^{-1}$ y el área de muestreo 6 es la única que no presentó daño por castores.

Al comparar la biomasa extraída en Pampa Guanaco con Lago Lynch, el valor estadístico (valor W) fue de 19 con $P=0,8764$ (alto), y si se compara con Timaukel el valor $\mathrm{W}$ fue 12 con $P=0,6623$ (alto). Al comparar Lago Lynch con Timaukel el valor $\mathrm{W}$ fue 13 y $P=0,2949$ (alto). En consecuencia, los resultados de la prueba de Wilcoxon indicaron que no hubo diferencias estadísticamente significativas en la biomasa leñosa de $N$. pumilio removida entre los tres sectores estudiados, por lo que el promedio de $14,9 \mathrm{t} \mathrm{ha}^{-1}$ de biomasa leñosa removida por castores en los bosques ribereños, es estadísticamente representativo para Tierra del Fuego. Las diferencias entre los promedios para cada sector dicen relación con la disponibilidad de N. pumilio. En Timaukel la presencia de otras especies arbóreas, como $N$. betuloides, característica de los sectores costeros de Tierra del Fuego (Vergara y Schlatter 2004) hace menos frecuente a $N$. pumilio, lo que explica una menor biomasa leñosa removida de esta especie.

De acuerdo a Johnston y Naiman (1990), en un bosque dominado por Populus tremuloides se determinó una biomasa leñosa removida por castores de $1,4 \mathrm{t} \mathrm{ha}^{-1} \mathrm{añon}^{-1}$, en un período de actividad de castores de seis años, lo que resulta en un total de 8,4 $\mathrm{tha}^{-1}$ de biomasa leñosa removida para ese período. Este valor es relativamente cercano a las $7,1 \mathrm{t} \mathrm{ha}^{-1}$ de biomasa leñosa de $N$. pumilio removida en Timaukel. Un factor en común entre el citado estudio y el valor de biomasa removida encontrado para Timaukel es que se trata de bosques mixtos, existiendo en ambos casos especies evitadas por los castores. Tal es el caso de Alnus rugosa en el estudio citado, y $N$. betuloides en el presente estudio. La mayor remoción del bosque dominado por $P$. tremuloides puede deberse a que su madera es más blanda comparada con $N$. pumilio.

En contraste, en Lago Lynch y Pampa Guanaco la biomasa removida es mucho mayor que las 8,4 tons/ha del estudio de Johnston y Naiman (1990). Esta diferencia sería consecuencia del dominio de bosques puros de $N$. pumilio en Pampa Guanaco y en Lago Lynch, sugiriendo que los castores consumen el recurso en forma proporcional a su disponibilidad.

Pérdidas de volumen bruto de N. pumilio. La pérdida de volumen bruto total de $N$. pumilio corresponde al volumen removido en los bosques ribereños más el volumen perdido por árboles muertos en zonas de inundación. Los promedios globales de tales volúmenes alcanzaron a 286,8 $\mathrm{m}^{3} \mathrm{ha}^{-1}$ (cuadro 3) y son representativos para N. pumilio en Tierra del Fuego, debido a que la comparación a través de la prueba de Wilcoxon indicó que no hubo diferencias estadísticamente significativas entre los tres sectores. Al comparar la pérdida de volumen bruto en Pampa Guanaco con Lago Lynch, el valor estadístico (valor W) es de 20 con $P=0.7550$ (alto), y si se compara con Timaukel el valor W es 14 con $P=0,9307$ (alto). Al comparar Lago Lynch con Timaukel el valor $\mathrm{W}$ es 15 y $P=0,4452$ (alto).

Metodológicamente se diferenció el cálculo de la pérdida de volumen por tala y el volumen perdido por árboles muertos en zonas de inundación. El volumen bruto perdido en zona de inundación alcanzó a 280,4 $\mathrm{m}^{3} \mathrm{ha}^{-1}(97,8 \%)$, mientras que la pérdida de volumen bruto por tala alcanzó a sólo $6,4 \mathrm{~m}^{3} \mathrm{ha}^{-1}(2,2 \%)$. Estos valores, que corresponden a promedios de volúmenes brutos, demostraron que la pérdida más importante de volumen de esta especie en Tierra del Fuego resultó de la muerte de individuos en zona de inundación, producto de la construcción de diques por parte de castores. 
Cuadro 3. Promedio de pérdida de volumen total por sector. Average of total volume loss by sampling sectors.

\begin{tabular}{lccc}
\hline Sector & $\begin{array}{c}\text { Pérdida de } \\
\text { volumen } \\
\text { bruto } \\
\text { promedio } \\
\left(\mathrm{m}^{3} \mathrm{ha}^{-1}\right)\end{array}$ & $\begin{array}{c}\text { Volumen } \\
\text { bruto } \\
\text { promedio } \\
\text { removido } \\
\text { por tala } \\
\left(\mathrm{m}^{3} \mathrm{ha}^{-1}\right)\end{array}$ & $\begin{array}{c}\text { Volumen bruto } \\
\text { promedio } \\
\text { removido } \\
\text { por tala }(\% \\
\text { respecto al } \\
\text { volumen } \\
\text { hipotético })\end{array}$ \\
\hline $\begin{array}{l}\text { Pampa Guanaco } \\
\text { Lago Lynch }\end{array}$ & 294,8 & 33,7 & 9,4 \\
Timaukel & 238,4 & 30,2 & 9,8 \\
\hline Promedio global & 286,8 & 18,7 & 8,0 \\
\hline
\end{tabular}

Son pocos los estudios que cuantifican el volumen perdido en los bosques ribereños afectados por castores, y en Chile sólo existe una prospección (Skewes et al. 1999), donde se afirma que las pérdidas en volumen llegan al $51,6 \%$ respecto al volumen total (dañado y no dañado), considerando la pérdida por inundación. En el presente estudio no se realizaron mediciones para estimar directamente las pérdidas en zona de inundación, como se hizo en los bosques ribereños. Sin embargo, considerando que el 100\% de los árboles en pie en zona de inundación están muertos, se puede considerar que el $100 \%$ del volumen en zona de inundación se perdió. Promediando el volumen que se pierde en bosques ribereños afectados sólo por remoción $(9,1 \%)$, se aprecia que el promedio entre pérdida de volumen en zona de inundación y pérdida de volumen en bosques ribereños es de 54,5\%. Esta pérdida en volumen se acerca a la estimada en el estudio anterior, y evidencia que más de la mitad de la existencia de volumen de los bosques de $N$. pumilio asociados a cursos de agua se ha perdido en Tierra del Fuego por efecto de los castores.

\section{DISCUSIÓN}

Influencia del castor en la dinámica de los bosques de N. pumilio. De continuar la situación actual, se advierte la posibilidad de un aumento en la superficie afectada por inundación y una consecuente mayor pérdida de volúmenes. Esto ocurre debido a sucesivas alteraciones del curso de agua original a través del tiempo, desviándose el flujo hacia bosques no afectados y por tanto incrementando las pérdidas.

El estudio indica que las actividades de castores se traducen en la muerte de aquella parte del bosque que alcanza a ser anegado cuando estos roedores construyen un dique, y en una importante alteración en la dinámica y estructura de los bosques aledaños. Estas alteraciones generan una brecha en las distribuciones diamétricas entre el bosque no afectado y el bosque afectado. Debido a la preferencia de los castores por diámetros menores, se presenta una fuerte disminución en la existencia de biomasa en las clases diamétricas menores en los bosques ribereños afectados. Esta preferencia por diámetros menores también compromete seriamente la generación de reemplazo al dificultar el establecimiento de la regeneración. De hecho, se constató en terreno una ausencia de regeneración en las zonas con árboles muertos por inundación, y que actualmente ya no están anegadas, encontrándose en su lugar un abundante estrato herbáceo. En estas zonas el bosque ya no existe como tal, desperfilando las microcuencas hidrográficas y creando praderas para el forrajeo de $L$. guanicoe.

Considerando que los bosques estudiados han sido afectados por la acción de los castores por un período relativamente corto respecto al ciclo de vida de $N$. pumilio, los resultados del presente estudio requieren un adecuado seguimiento y monitoreo a través del tiempo para analizar la evolución de los bosques dañados. No obstante, el efecto de $C$. canadensis ya es importante tanto en número de árboles afectados, así como en la pérdida de biomasa y volumen de $N$. pumilio, lo que demanda urgentes medidas de manejo y una respuesta institucional que en general se aprecia lenta frente a las diversas especies invasoras presentes en el país (Iriarte et al. 2005). Estas medidas deben focalizarse a la recuperación del bosque alterado, con énfasis en los diámetros menores, junto con medidas directas para la disminución de la población, e inclusive la erradicación de $C$. canadensis de Tierra del Fuego. Esto reviste particular importancia por dos razones fundamentales: en primer lugar las zonas alteradas corresponden a bosques de protección de acuerdo a la legislación vigente, a partir de la Ley de Bosques del año 1931, por cumplir una importante función protectora del régimen hídrico. En segundo lugar, forman parte de una extensa área protegida privada que administra Wildlife Conservation Society (WCS), organismo norteamericano que ha establecido el parque Karukinka, con cerca de 270 mil ha, principalmente con fines científicos y de conservación de la biodiversidad.

Al daño directo del castor cabe agregar que para los bosques de Magallanes se han documentado superficies de hasta 100 ha devastadas por efecto del viento (Le Quesne 2002) y que en la zona de estudio N. pumilio presenta agentes de biodegradación, principalmente hongos lignívoros causantes de la pudrición blanca y café (CONAF/ GTZ 1998), lo que los hace aún más vulnerables.

Comparación del castor en su ecosistema exótico con su hábitat nativo. Existen estudios comparativos entre la influencia del castor en ecosistemas exóticos con respecto a su hábitat natural. Uno de los estudios más recientes realizado por Anderson et al. (2006b) concluye que el impacto inicial fue muy similar. En ambos casos los castores modificaron la dinámica de los bosques y cambiaron la composición de especies, incluyendo abundantes estratos herbáceos, que en Norteamérica constituyen comunidades únicas. Cuando en Norteamérica los diques colapsan y el 
estanque se drena, deja un sustrato rico en nutrientes para la colonización de especies pioneras de plantas y animales, formando el llamado "prado del castor" después de un período aproximado de 15 años de abandono (D’eon et al. 1995).

Sin embargo, estudios en Chile demuestran que el castor está produciendo daños a largo plazo. Por una parte, las alteraciones prácticamente eliminan la regeneración boscosa, situación que no ocurre en los bosques pertenecientes a la distribución natural del castor, ya que los bosques subantárticos no están adaptados para regenerar bajo este tipo de disturbios (Anderson et al. 2006b). Los mismos autores señalan que el carácter silvestre del ecosistema subantártico está amenazado por la alteración al ambiente y la invasión de plantas introducidas, que es favorecida por la acción de los castores, y tampoco se han encontrado comunidades únicas, posterior al daño del castor, como sucede en Norteamérica. Frente a esta situación se requiere una acción institucional coordinada entre los numerosos organismos de Chile y Argentina involucrados con el tema de especies invasoras.

\section{AGRADECIMIENTOS}

Los autores desean expresar sus sinceros agradecimientos a WCS por su apoyo durante la etapa de terreno y las facilidades para usar su información cartográfica. También agradecen a la Corporación Nacional Forestal por el apoyo financiero y al Comité Editorial y jueces de la Revista Bosque por los valiosos comentarios que permitieron enriquecer versiones preliminares del manuscrito.

\section{REFERENCIAS}

Anderson CB, R Rozzi, JC Torres-Murúa, SM McGehee, MF Sherriffs, E Schüttler, AD Rosemond. 2006a. Exotic vertebrate fauna in the remote and pristine sub-Antarctic Cape Horn Archipelago, Chile. Biodiversity and Conservation 10: 3295-3313.

Anderson CB, CR Griffith, AD Rosemond, R Rozzi, O Dollenz. 2006b. The effects of invasive North American beavers on riparian communities in Cape Horn, Chile: Do exotic beavers engineer differently in sub-Antarctic ecosystems? Biological Conservation 128: 467-474.

Barnes WJ, E Dibble. 1988. The effects of beaver in riverbank forest succession. Canadian Journal of Botany 66: 40-44.

Briones C, R Schlatter, A Wolodarsky, C Venegas. 2001. Clasificación ambiental para hábitats de Castor canadensis (Kuhl 1820, Rodentia) de acuerdo a las características de cuencas en el sector de Tierra del Fuego. Anales Instituto Patagonia, Serie Ciencias Naturales 29: 75-93.

CONAF/GTZ. 1998. Experiencia silvicultural del bosque nativo de Chile. Santiago, Chile. Pub. Lo Castillo. 420 p.

Crooks JA. 2002. Characterizing ecosystem-level consequences of biological invasion: the role of ecosystem engineers. Oikos 97: 153-166.
D'eon RG, R Lapointe, N Bosnick, JC Davies, B MacLean, WR Watt, RG Wilson. 1995. The Beaver Handbook: A Guide to Understanding and Coping with Beaver Activity. Northeast Science and Technology, Ontario Ministry of Natural Resources. Timmins, ON. 76 p.

Fryxell JM, CM Doucet. 1993. Diet choice and the functional response of beavers. Ecology 70: 310-316.

Gajardo R. 1993. La vegetación natural de Chile. Clasificación y distribución geográfica. Santiago, Chile. Editorial Universitaria. $165 \mathrm{p}$.

Gallant D, CH Bérubé, E Tremblay, L Vasseur. 2004. An extensive study of the foraging ecology of beavers (Castor canadensis) in relation to habitat quality. Canadian Journal of Zoology 82: 922-923.

Härkönen S. 1999. Forest damage caused by the Canadian beaver (Castor canadensis) in South Savo, Finland. Silva Fennica 33: 247-259.

Iriarte, A. 2000. Impacto de la fauna silvestre sobre la flora nativa de Chile. In Baldini A, L Pancel eds. Agentes de daño en el bosque nativo. Santiago, Chile. Editorial Universitaria. p. 319-350.

Iriarte A, G Lobos, F Jaksic. 2005. Invasive vertebrate species in Chile and their control and monitoring by governmental agencies. Revista Chilena de Historia Natural 78: 143-154.

Jaksic FM, JA Iriarte, JE Jiménez, DR Martínez. 2002. Invaders without frontiers: cross-border invasion of exotic mammals. Biological Invasion 4: 157-173.

Johnston CA, RJ Naiman. 1987. Boundary dynamics at the aquatic-terrestrial interface: the influence of beaver and geomorphology. Landscape Ecology 1: 47-57.

Johnston CA, RJ Naiman. 1990. Browse selection by beaver: effects on riparian forest composition. Canadian Journal of Forest Research 20: 1036-1043.

Le Quesne C. 2002. Bioecología del bosque nativo chileno. In Baldini A, L Pancel eds. Agentes de daño en el bosque nativo. Santiago, Chile. Editorial Universitaria. p. 19-44.

Lizarralde M. 1993. Current status of the introduced beaver (Castor canadensis) population in Tierra del Fuego, Argentina. Ambio 22: 351-358.

Lizarralde M, J Escobar, G Deferrari. 2004. Invader species in Argentina: a review about the beaver (Castor canadensis) population situation on Tierra del Fuego ecosystems. Interciencia 29: 352-355.

Magni C. 1995. Acumulación de biomasa y nutrientes en un bosque mixto natural de lenga (Nothofagus pumilio) y coihue de Magallanes (Nothofagus betuloides) en la XII Región. Tesis Ingeniero Forestal. Santiago, Chile. Universidad de Chile, Facultad de Ciencias Agrarias y Forestales. 93 p.

Martínez G, V Lencinas, J Escobar, P Quiroga, L Malmierca, M Lizarralde. 2006. Understory succession in areas of Nothofagus forests in Tierra del Fuego (Argentina) affected by Castor canadensis. Journal of Applied Vegetation Science 9: 143-154.

Mella J, M Díaz, B Saavedra, P Sabat, C Smith, C Veloso, AM Humaña. 1995. Castores. Comité Científico Proyecto Río Cóndor. Estudio de Línea de Base Informe del Subproyecto 94-14 Castores. 39 p.

Naiman R, C Johnston, J Kelley. 1988. Alteration of North American streams by beaver. Bioscience 38: 753-761. 
Nummi P. 2006. NOBANIS Invasive Alien Species Fact Sheet Castor canadensis. Online Database of the North European and Baltic Network on Invasive Alien Species - NOBANIS. Consultado 21 abr. 2008. Disponible en http://www.nobanis.org.

Olave R, O Skewes. 2000. Distribución del castor (Castor canadensis) en Tierra del Fuego, XII Región. Bosque Nativo (Chile) 25: 16-21.

Pulido FJ, B Díaz, P Martínez. 2000. Incidencia del ramoneo del guanaco (Lama guanicoe) sobre la regeneración temprana en bosques de lenga (Nothofagus pumilio) de Tierra del Fuego, Argentina. Investigación Agraria, Sistemas y Recursos Forestales 9(2): 381-394.

Rius F, F Barón, L Parras, E Sánchez. 1999. Bioestadística: Métodos y Aplicaciones ( $3^{\mathrm{a}}$ edición). Málaga, España. Universidad de Málaga. 322 p.

Rosell F, O Bozsér, P Collen, H Parker. 2005. Ecological impact of beavers Castor fiber and Castor canadensis and their ability to modify ecosystems. Mammal Review 35: 248-276.

Schlatter R, P Vergara, M Briones. 2002. El canquén (Chloephaga poliocephala: Anatidae) en bosques de Tierra del Fuego: distribución y depredadores. Anales Instituto de la Patagonia, Serie Ciencias Naturales 30: 61-66.

Schmith H, A Urzúa. 1982. Transformación y manejo de los bosques de lenga en Magallanes. Santiago, Chile, Ciencias
Agrícolas $\mathrm{N}^{\circ} 11$, Universidad de Chile, Facultad de Ciencias Agrarias y Forestales. $62 \mathrm{p}$.

Siefeld W, C Venegas. 1980. Poblamiento e impacto ambiental de Castor canadensis en Isla Ambarino, Chile. Anales Instituto Patagonia 11: 247-257.

Skewes O, F González, L Rubilar, OM Quezada, RR Olave, VR Vargas, AC Ávila. 1999. Investigación, aprovechamiento y control del castor en islas Tierra del Fuego y Navarino. Punta Arenas, Chile. Servicio de Gobierno Regional XII Región, Magallanes y Antártica Chilena. 185 p.

Skewes O, F González, R Olave, A Ávila, V Vargas, P Paulsen, H König. 2006. Abundance and distribution of American beaver, Castor canadensis (Kuhl 1820), in Tierra del Fuego and Navarino islands, Chile. European Journal of Wildlife Research 52: 292-296.

Vergara P, R Schlatter. 2004. Magallanic woodpecker (Campephilus magallanicus) abundance and foraging in Tierra del Fuego, Chile. Journal of Ornithology 145: 343-351.

Wallem PK, C Jones, P Marquet, F Jaksic. 2007. Identificación de los mecanismos subyacentes a la invasión de Castor canadensis (Rodentia) en el archipiélago de Tierra del Fuego, Chile. Revista Chilena de Historia Natural 80:309-325.

Wright JP, CG Jones, AS Flecker. 2002. An ecosystem engineer, the beaver, increases species richness at the landscape scale. Oecología 132: 96-101. 